\title{
Further Insight into Mechanisms of Ventricular Tachycardia from the Clinical Electrophysiology Laboratory
}

\author{
FRED MORADY, M.D. \\ From the Division of Cardiology, Department of Internal Medicine, The University of Michigan Medical Center, \\ Ann Arbor, Michigan
}

Sustained, monomorphic ventricular tachycardia (VT) in patients with prior myocardial infarction is usually caused by reentry (Wellens, et al., 1976; Josephson, et al., 1985; de Bakker, et al., 1988; Downar, et al., 1988), and several different lines of evidence from experimental and clinical studies have implicated a zone of slow conduction as being a critical component of the reentrant circuit (El-Sherif, et al., 1983; Gessman, et al., 1983; Gallagher, et al., 1985; Frank, et al., 1987; Stevenson, et al., 1987; Kay, et al., 1988; Morady, et al., 1988). In a study published in this issue of the Journal of Cardiovascular Electrophysiology, Fitzgerald et al. (1991) present evidence that a single area of slow conduction may participate in the reentrant circuits of two or three different VTs. This elegant study provides new insight into mechanisms of VT and also serves as an excellent example of the value of carefully recording and analyzing intracardiac electrograms in the clinical electrophysiology laboratory.

\section{Isolated Diastolic Potentials}

Fitzgerald et al. focused their attention on isolated diastolic potentials recorded in bipolar fashion by closely-spaced electrodes against the endocardial surface during VT in patients with prior myocardial infarction. Experimental studies have demonstrated that diastolic potentials during VT may originate in regions of slow conduction (El-Sherif, et al., 1983; Gessman, et al., 1983). However, patients with prior myocardial infarc-

J Cardiovasc Electrophysiol, Vol. 2, pp. 207-214, June 1991.

Address for correspondence: Fred Morady, M.D., Division of Cardiology, The University of Michigan Medical Center, 1500 East Medical Center Drive, B1 F245, Ann Arbor, MI 48109-0022. Fax: 313-936-7641.

Manuscript received 4 March 1991; Accepted for publication 8 March 1991. tion may have more than one region of slow conduction, and not all of these regions of slow conduction would have to be critical components of a reentrant circuit. Therefore, the presence of an isolated diastolic potential during VT by itself would not necessarily identify a zone of slow conduction that participates in the reentrant circuit.

The determination of whether the site at which a diastolic potential is recorded is part of the reentrant circuit requires several pacing maneuvers, as demonstrated in a prior study by Fitzgerald et al. (1988). If the diastolic potential can be dissociated from the ventricular electrogram of the VT by ventricular pacing during VT or during the initiation or termination of VT, this would indicate that the site at which the diastolic potential was recorded was not a critical component of the reentrant circuit, but instead an "innocent bystander." However, if the diastolic potential maintained a constant relationship to the ventricular electrogram during initiation and pertubations of the VT, this would be evidence (but not definitive proof) that the recording site was part of the reentrant circuit. Because it is conceivable that a region of slow conduction that is an innocent bystander could be activated in such a way as to maintain a fixed relationship to the next VT complex, there may be no way to prove that a site at which a diastolic potential is recorded is actually part of the reentrant circuit of the VT.

Nevertheless, in a prior study, Fitzgerald et al. (1988) demonstrated the clinical utility of the isolated diastolic potential as a guide for selection of target sites for catheter ablation of VT. Seven of seven VTs were successfully ablated when shocks were delivered to sites at which isolated diastolic potentials that could not be dissociated from the VT were recorded. This suggests that the sites were critical components or at least close to critical components of the reentrant circuit. 


\section{Concealed Entrainment}

Another approach to identifying a region of slow conduction that may participate in the VT reentrant circuit involves analysis of the response to ventricular pacing during VT. Entrainment of VT by ventricular pacing at a site that is not part of the reentrant circuit results in fusion of the QRS complexes because of simultaneous ventricular activation at the pacing site and at the exit site of the reentrant circuit (Waldo, et al., 1990). If the pacing site is within a region of slow conduction that is bounded by arcs of conduction block, pacing during VT may result in acceleration of the VT to the pacing rate, a long stimulus-QRS interval, and no change in the QRS morphology compared to the VT (Fig. 1). This phenomena, referred to as "concealed entrainment" because there is no QRS fusion during VT, results from block in the antidromic direction away from the pacing site and slow conduction in the orthodromic direction (Morady, et al., 1988; Waldo, et al., 1990; Morady, et al., 1991). Because global ventricular activation is delayed until the wave of depolarization exits from the region of slow conduction, the stimulus-QRS interval during concealed entrainment may be as long as 400 msec (Morady, et al., 1991).

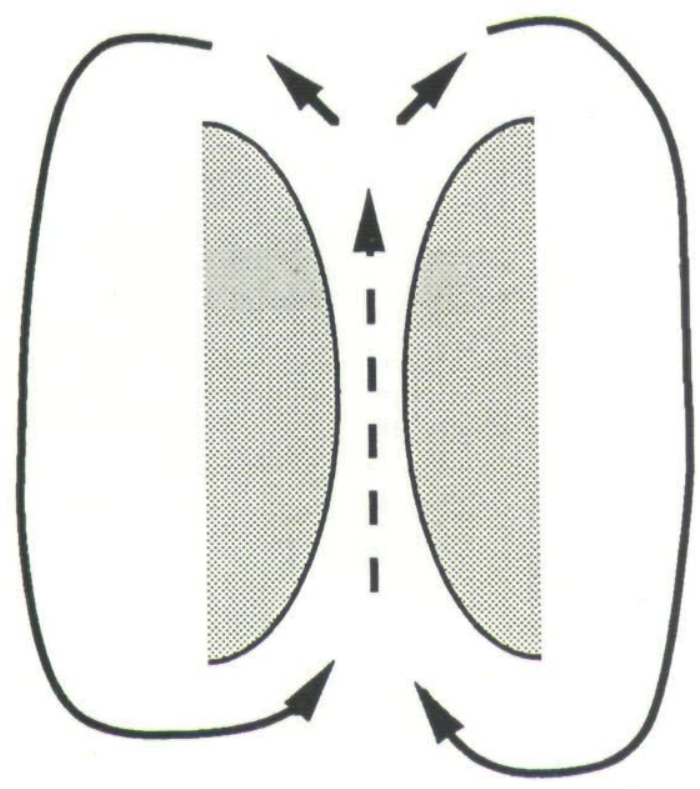

In their study, Fitzgerald et al. (1991) did not pace from the sites at which isolated diastolic potentials were recorded during VT. The demonstration of concealed entrainment at the sites at which diastolic potentials were recorded would have provided additional evidence that these sites were in a region of slow conduction which was part of the VT reentrant circuit. An example of this is shown in Figures 2A-D. A man with coronary artery disease and a history of myocardial infarction had frequent episodes of VT that had a left bundle branch block morphology and a rate of approximately 120 beats/min (Fig. 2A). An isolated diastolic potential was recorded during VT at the basal septum of the left ventricle (site 4-6, Fig. 2B). The diastolic potential could not be dissociated from the VT by various pacing maneuvers. Pacing at this site at a cycle length of $400 \mathrm{msec}$ during VT resulted in concealed entrainment: the VT accelerated to the pacing cycle length, the stimulus-QRS interval was $240 \mathrm{msec}$, and the QRS morphology during pacing was identical to the VT QRS morphology (Fig. 2C).

These observations support the conclusion that the site at which the isolated diastolic potential was recorded was indeed within a zone of slow conduction that participated in the reentrant

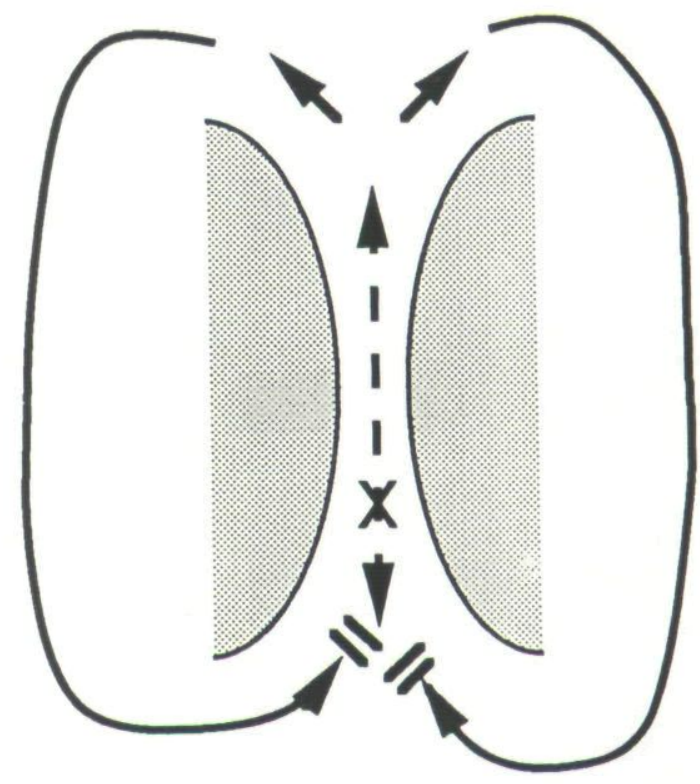

Figure 1. Schematic representation of concealed entrainment of a ventricular tachycardia (VT) reentrant circuit that has a figure-8 configuration. On the left is shown a zone of slow conduction (dashed line) bounded by two arcs of conduction block (shaded areas). Pacing at site $X$ within the zone of slow conduction (shown on the right) at a rate faster than that of the VT might result in collision in the antidromic direction with the reentrant wave front of the prior VT cycle, and slow conduction in the orthodromic direction towards the exit site from the zone of slow conduction. This would result in concealed entrainment, in which the VT accelerates to the pacing rate, there is a long stimulus-QRS interval (> 100 msec), and the QRS complexes during pacing and VT are identical. 
I

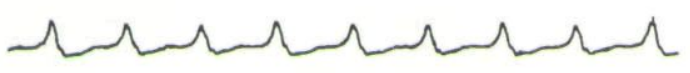

" lasasa

II hashah

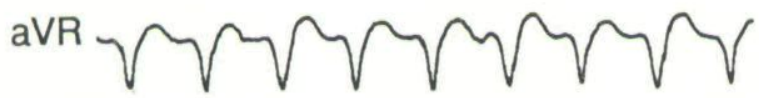

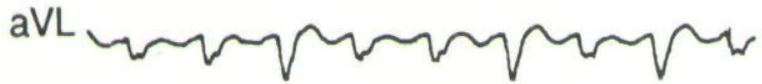

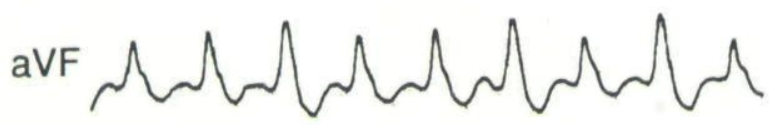

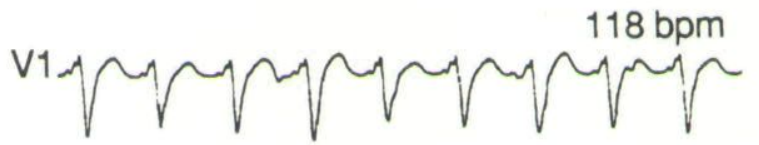

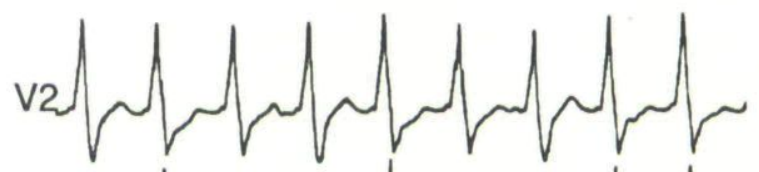
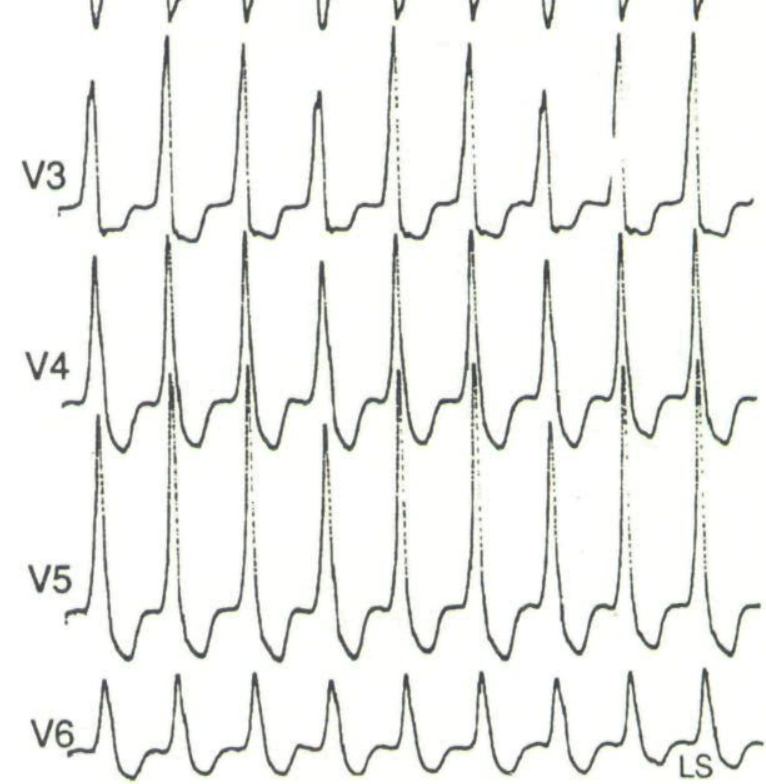

Figure 2A. Ventricular Tachycardia, rate 118 bpm, which has a left bundle branch block morphology and an inferior axis.

circuit. An alternative possibility is that the pacing site was near the exit site of the VT and that the long stimulus-QRS interval simply was a result of a long stimulus latency instead of concealed entrainment. However, this possibility can be ruled out by comparing the response to pacing during VT with the response to pacing at the same site during sinus rhythm. Pacing in the setting of VT resulted in a stimulus-QRS interval of $240 \mathrm{msec}$ and no change in the QRS morphology. In marked contrast, pacing at the same site during sinus rhythm resulted in a much shorter stimulus-QRS interval of $80 \mathrm{msec}$ and a dramatic change in the QRS morphology compared to the VT (Fig. 2D). This observation provides strong evidence that the long stimulusQRS interval during VT actually was a result of concealed entrainment. Of note is that the different QRS morphology during pacing in the setting of sinus rhythm also provides strong evidence that the zone of slow conduction was bounded by arcs of functional conduction block that were present during VT but not during sinus rhythm.
Aside from the mechanistic implications of these types of observations, they clearly also have clinical utility. Localization of a region of slow conduction that participates in the reentrant circuit may identify an excellent site for catheter ablation of VT (Frank, et al., 1987; Stevenson, et al., 1987; Morady, et al., 1988, 1991). For example, the VT that is shown in Figure 2 was successfully ablated by a 200 -joule DC shock delivered through an electrode catheter positioned at the site at which the isolated diastolic potential and concealed entrainment were demonstrated.

\section{Termination of VT by Subthreshold Stimuli}

Although the ability to demonstrate concealed entrainment by pacing at a site at which an isolated diastolic potential is present during VT provides additional evidence that the site is within a region of slow conduction, it would still be possible for the region of slow conduction to not be a critical component of the reentrant circuit. Pacing within a region of slow conduction, that is connected to the reentrant circuit but which is not 


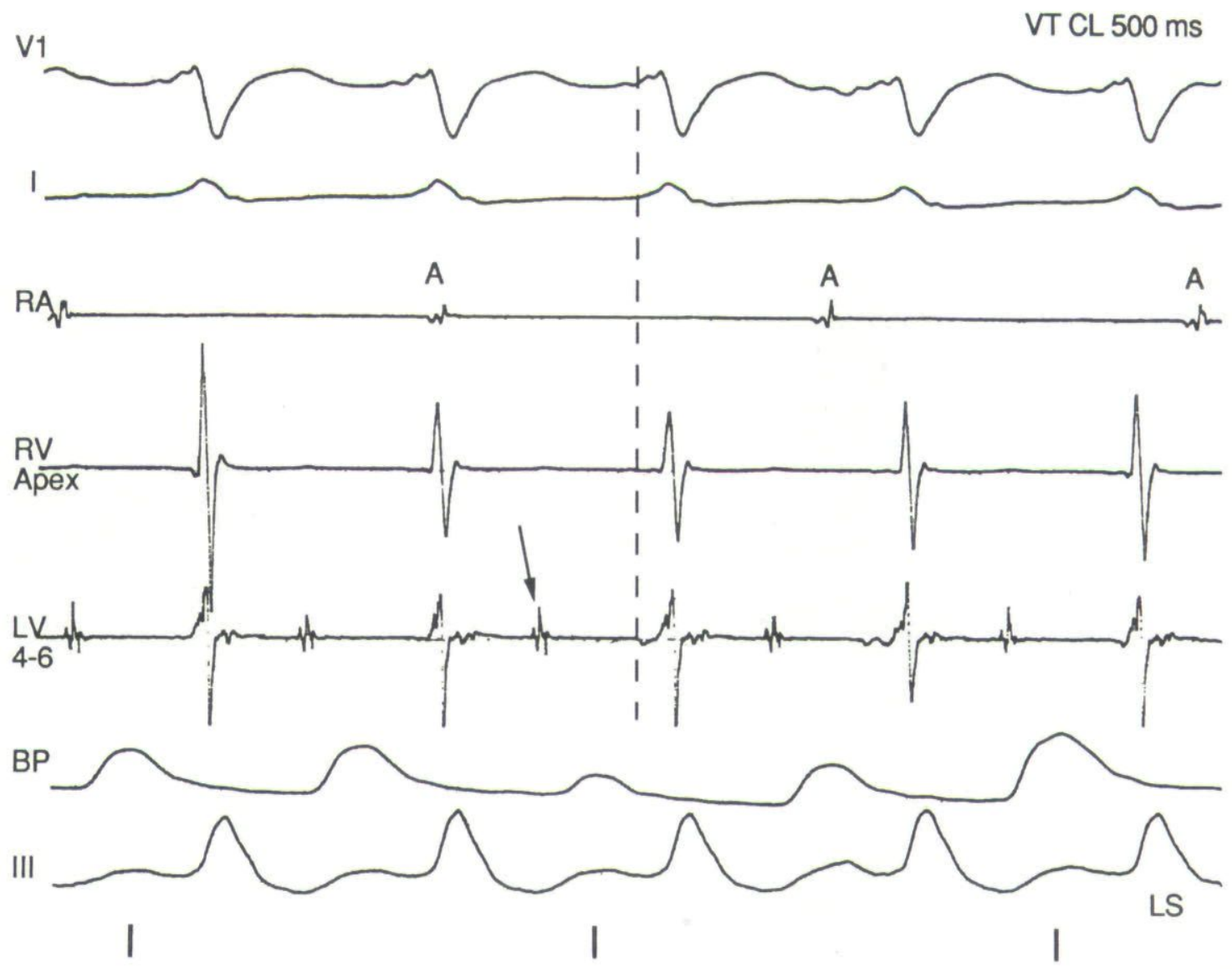

Figure 2B. An isolated diastolic potential (arrow) was recorded at the basal septum of the left ventricle (LV 4-6). Also shown are leads VI, I, right atrial (RA) and right ventricular $(R V)$ electrograms, a blood pressure recording (BP), and lead III. The time lines indicate 1-second intervals.

critical to the maintenance of reentry might also result in concealed entrainment (Fig. 3). This may be one of the explanations for why the success rate of catheter ablation is only a relatively modest $55 \%$ when electric shocks are delivered to sites of concealed entrainment (Morady, et al., 1991).

Perhaps the best evidence obtainable in the clinical electrophysiology laboratory that a site at which there is concealed entrainment and/or isolated diastolic potentials is a critical component of the reentrant circuit is the ability to terminate the VT with a subthreshold premature stimulus applied at that site. Ruffy et al. (1983; Ruffy, 1990) and Podczeck et al. (1988) demonstrated that a premature stimulus that resulted in local capture but which did not propagate to the ventricles could reproducibly terminate VT when delivered within a zone of slow conduction. It is presumed that local capture within the region of slow conduction does not result in global ventricular capture because of the arcs of conduction block that bound the slow zone. A nonpropagated stimulus might terminate VT if it is premature enough to impinge on the refractory period of the slow zone in the orthodromic direction and if there is collision of wave fronts in the antidromic direction away from the pacing site. Termination of VT by a nonpropagated local capture within the zone of slow conduction would be possible only if the stimulation site was critical to the maintenance of reentry. Theoretically, termination of VT by a subthreshold stimulus identifies a vulnerable portion of the reentrant circuit and an ideal site for catheter ablation of the VT.

\section{Pleomorphic Ventricular Tachycardia}

Patients with coronary artery disease and VT often have morphologically distinct (pleomorphic) VTs (Josephson, et al., 1979). Theoretically, multiple morphologies of VT could be a manifestation of different exit sites from a single 

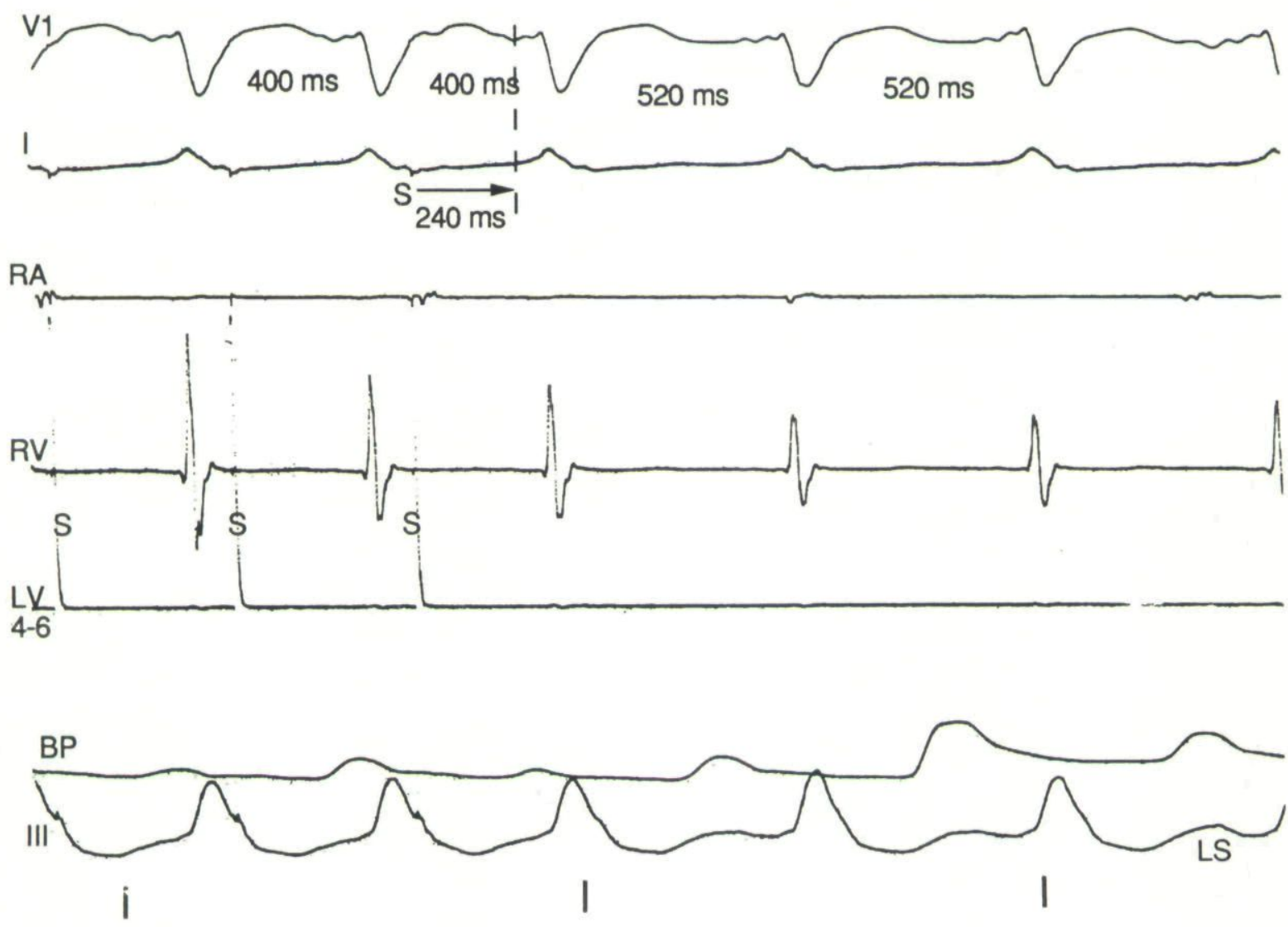

Figure 2C. Pacing at a cycle length of $400 \mathrm{~ms}$ during VT at site LV 4-6 resulted in concealed entrainment. Shown are the last three pacing stimuli (S) of a 10-beat train. The stimulus-QRS interval is $240 \mathrm{~ms}$ and the QRS complexes during pacing and during VT are identical.

reentrant circuit or could arise from anatomically separate reentrant circuits. Prior mapping studies performed in the electrophysiology laboratory and in the operating room have provided evidence that morphologically distinct VTs often arise from the same circuit or from circuits that are within 1 to $2 \mathrm{~cm}$ of each other, and that only a minority of patients have disparate sites of VT origin located more than $5 \mathrm{~cm}$ from one another (Josephson, et al., 1980; Miller, et al., 1984). Although some investigators have speculated that morphologically distinct VTs might share the same region of slow conduction (Kuck, et al., 1989), convincing evidence to support this possibility has been lacking.

The astute observations of Fitzgerald et al. provide new insight into possible mechanisms of pleomorphic VT by demonstrating that a single region of slow conduction may indeed participate in the reentrant circuits of two or even three morphologically distinct VTs. Recordings obtained at a single site in the left ventricle demonstrated the presence of an isolated diastolic potential that could not be dissociated from the VT during two or three different forms of VT. Because these recordings were performed in bipolar fashion using closely-spaced electrodes, it can be presumed that the diastolic potentials recorded at this single catheter position during different VTs were arising from the same relatively small region of myocardium. These observations provide convincing evidence that the reentrant circuits of morphologically distinct VTs may share the same region of slow conduction.

Carrying their analysis one step further, based on the morphology and timing of the diastolic potentials, Fitzgerald et al. propose three different mechanisms by which morphologically distinct VTs might share the same region of slow conduction. The VTs may utilize varying lengths of the same region of slow conduction, which is activated in the same direction during both VTs. A second possibility is that the shared region of slow conduction is traversed in opposite directions during the different VTs. Thirdly, the region of slow conduction may be similar in length and 
Pacing During VT

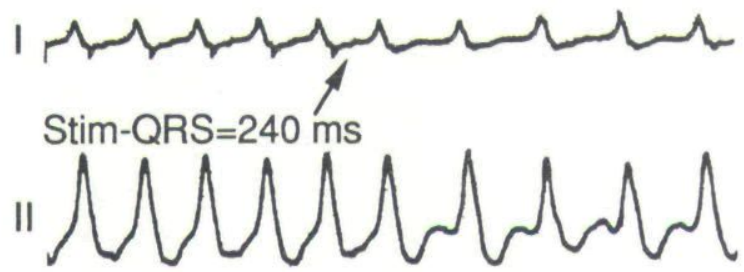

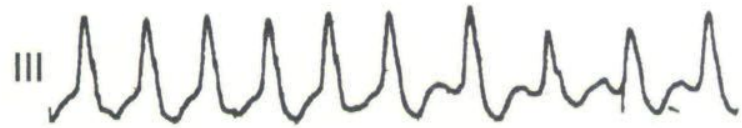

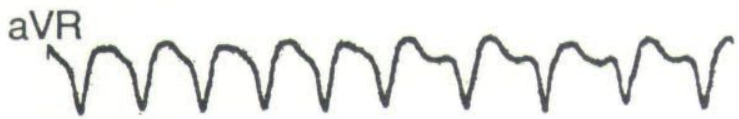

aVL
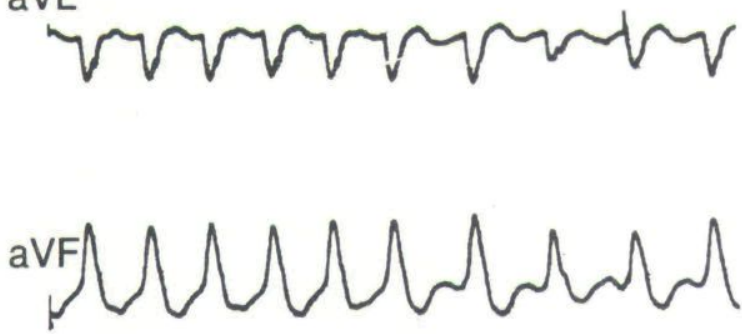

Pacing During Sinus Rhythym
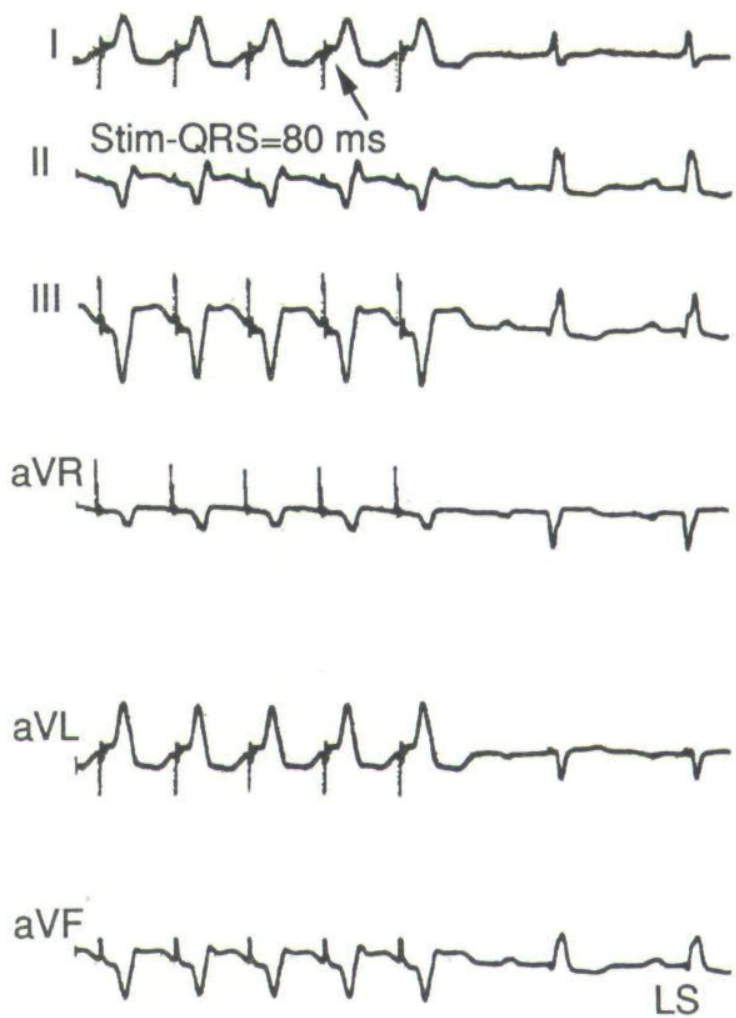

Figure 2D. A comparison of the response to pacing at site LV 4-6 during VT and during sinus rhythm. The stimulus-QRS interval was much shorter and the QRS configuration was very different than the VT when pacing in the setting of sinus rhythm. See text for explanation.

activated in the same direction during both VTs, and the pleomorphism may be related to changes in ventricular activation outside the region of slow conduction. These three possibilities provide plausible explanations for the three different patterns of changes in diastolic potential morphology and timing observed in their study during transition from one VT to another.

A particularly intriguing pattern of diastolic potentials was observed during the VTs illustrated in Figure 7 of Fitzgerald et al.'s article. Two diastolic potentials were present during sinus rhythm, pacing, and two morphologically distinct VTs. Of note is that one of the diastolic potentials always maintained the same morphology but varied in its timing, whereas the second diastolic potential varied in morphology and timing. In a masterful display of deductive reasoning and ingenuity, the authors provide a detailed explanation for their observations in their Figure 11, which demonstrates how a region of slow conduction might be activated differently during pleomorphic VTs.
To explain the presence of a diastolic potential that maintains the same configuration but changes in its timing during two morphologically distinct VTs, Fitzgerald et al. propose the explanation illustrated in their Figure 10, in which two morphologically distinct VTs with different rates share the same region of slow conduction but differ in the location of their exit sites. However, the model proposed in Figure 10 leaves some questions unanswered. For example, if one assumes that the exit site of the more rapid VT has a longer refractory period than the exit site of the slower VT, this would explain why a critical degree of pacing-induced shortening of the interval between consecutive diastolic potentials during the faster VT might result in block at the exit site of the faster VT and transition to the slower VT (Fig. 10, panel D). However, why would the exit site of the faster VT not become again excitable during the next cycle? At a minimum, one would have to postulate repeated concealed retrograde penetration at the exit site of the faster VT. But then, why would a slight 


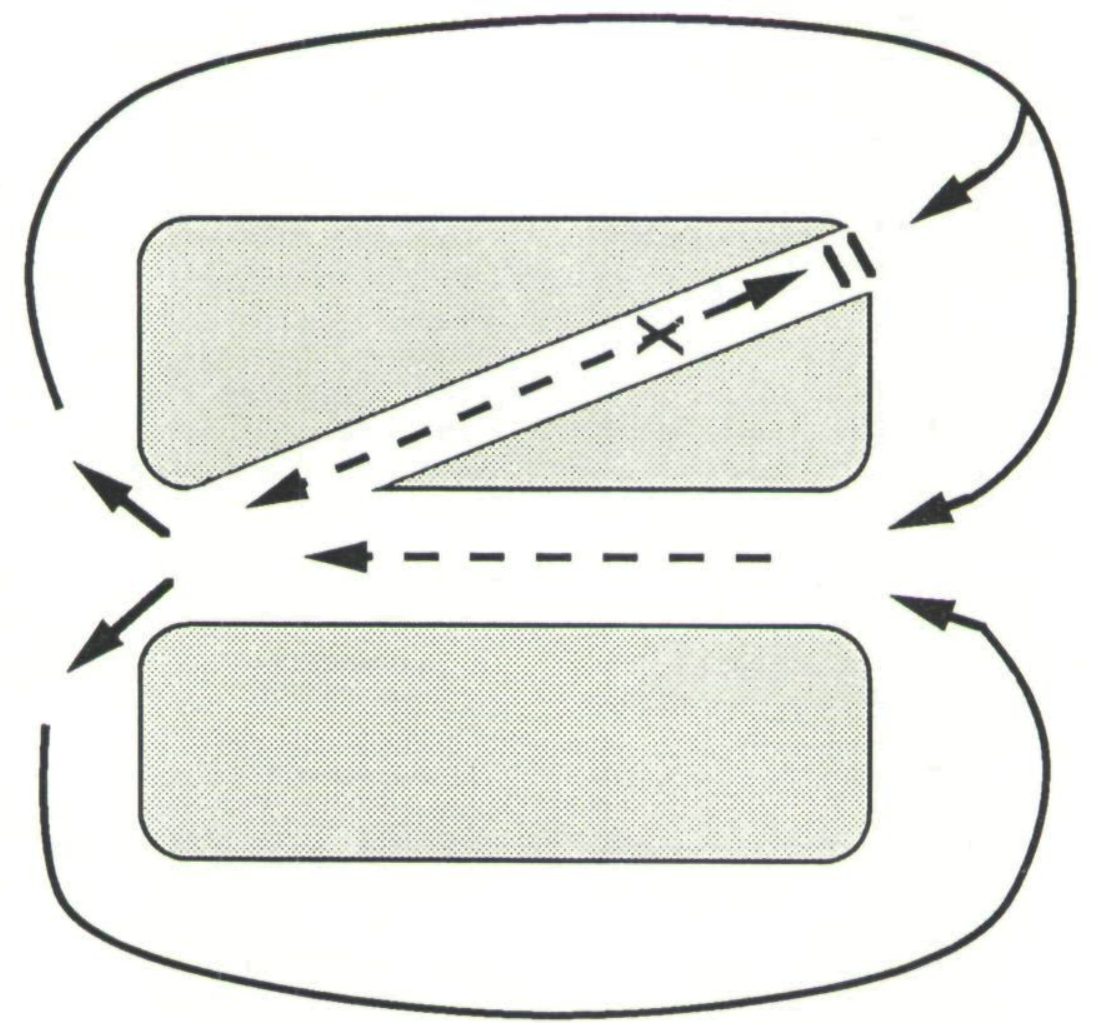

Figure 3. A schematic representation of a reentrant circuit, which includes an alternate slow pathway not critical for the maintenance of reentry. Pacing at site $X$ within this alternate slow pathway might result in concealed entrainment, however, reentry could continue even if this alternate pathway were to be ablated.

increase in the interval between consecutive diastolic potentials (from 370 to $380 \mathrm{msec}$; Fig. 2 , panel B) result in a switch back to the faster VT? These problems with the model proposed in Figure 10 might be resolved by postulating that the only portion of the reentrant circuit common to both VTs is the portion in the immediate vicinity of the recording electrodes. Extrastimuli could then result in termination of one VT and induction of the other one and the morphology of the diastolic potential would maintain a similar morphology but vary in its timing during the two VTs.

\section{Clinical Implications and Questions Remaining to be Answered}

An important therapeutic implication of the findings of Fitzgerald et al. is that pleomorphic VTs may be amenable to ablation by targeting a single, relatively small area of a region of slow conduction critical to the reentrant circuit of each of the morphologically distinct VTs. This is particularly relevant when using catheter ablation techniques to treat VT. In the past, patients with only one form of monomorphic VT were thought to be the best candidates for catheter ablation, since the presence of pleomorphic VT might necessitate ablation at multiple sites, decreasing the possibility of a successful outcome and increasing the risk of complications. But Fitzgerald et al. have demonstrated that patients with pleomorphic VT may be ideal candidates for catheter ablation if it can be demonstrated that the pleomorphic VTs share a common region of slow conduction.

It remains to be determined how often pleomorphic VTs utilize the same region of slow conduction. The best mapping technique for demonstrating that a region of slow conduction is critical to the maintenance of reentry also remains to be established. It is likely that these issues will be resolved by studies performed in the experimental laboratory, operating room, and clinical electrophysiology laboratory. Fitzgerald et al. should be complimented for advancing our 
understanding of VT and also for reminding us of the tremendous appeal of cardiac electrophysiology to anyone who enjoys brain teasers. Analyzing a coronary angiogram could never be this much fun!

Acknowledgment: The author is grateful for the expert assistance of Marion Maguire in the preparation of this manuscript.

\section{References}

de Bakker JMT, van Capelle FJL, Janse MJ, Wilde AAM, et al: Reentry as a cause of ventricular tachycardia in patients with chronic ischemic heart disease: Electrophysiologic and anatomic correlation. Circulation 1988;77:589-606.

Downar E, Harris L, Mickleborough LL, Shaikh N, et al: Endocardial mapping of ventricular tachycardia in the intact human ventricle: Evidence for reentrant mechanisms. J Am Coll Cardiol 1988;11:783-791.

El-Sherif N, Mehra R, Gough WB, Zeiler RH: Reentrant ventricular arrhythmias in the late myocardial infarction period. Interruption of reentrant circuits by cryothermal techniques. Circulation 1983;68:644-656.

Fitzgerald DM, Friday KJ, Yeung Lai Wah JA, Lazzara R, et al: Electrogram patterns predicting successful catheter ablation of ventricular tachycardia. Circulation 1988;77:806-814.

Fitzgerald DM, Friday KJ, Yeung-Lai-Wah JA, Bowman AJ, et al: Myocardial regions of slow conduction participating in the reentrant circuit of multiple ventricular tachycardias: Report on ten patients. J Cardiovasc Electrophysiol 1991;2:193-206.

Frank R, Tonet JL, Kounde S, Farenq G, et al: Localization of the area of slow conduction during ventricular tachycardia. In: Brugada P, Wellens HJJ, eds. Cardiac Arrhythmias: Where To Go From Here? Futura Publishing Company, Inc., Mt. Kisco, 1987; p. 191-208.

Gallagher JD, Del Rossi AJ, Fernandez J, Maranhao $\mathrm{V}$, et al: Cryothermal mapping of recurrent ventricular tachycardia in man. Circulation 1985;71:733-739.

Gessman LJ, Agarwal JB, Endo T, Helfant RH: Localization and mechanism of ventricular tachycardia by ice mapping 1 week after the onset of myocardial infarction in dogs. Circulation 1983;68:657-663.

Josephson ME, Buxton AE, Marchlinski FE, Doherty JU, et al: Sustained ventricular tachycardia in coronary artery disease-Evidence for reentrant mechanism. In: Zipes DP, Jalife J, eds. Cardiac Electrophysiology and Arrhythmias. Grune \& Stratton, Inc., New York, 1985; p.409.
Josephson ME, Horowitz LN, Farshidi A, Spielman SR, et al: Recurrent sustained ventricular tachycardia: 4. Pleomorphism. Circulation 1979;59:459-468.

Josephson ME, Horowitz LN, Spielman SR, Greenspan AM, et al: Comparison of endocardial catheter mapping with intraoperative mapping of ventricular tachycardia. Circulation 1980;61:395-404.

Kay GN, Epstein AE, Plumb VJ: Region of slow conduction in sustained ventricular tachycardia: Direct endocardial recordings and functional characterization in humans. J Am Coll Cardiol 1988;11:109-116.

Kuck K-H, Schluter M, Kunze K-P, Geiger M: Pleomorphic ventricular tachycardia: Demonstration of conduction reversal within the reentry circuit. PACE 1989;12:1055-1064.

Miller JM, Kienzle MG, Harken AH, Josephson ME: Morphologically distinct sustained ventricular tachycardias in coronary artery disease: Significance and surgical results. J Am Coll Cardiol 1984;4:10731079.

Morady F, Frank R, Kou WH, Tonet JL, et al: Identification and catheter ablation of a zone of slow conduction in the reentrant circuit of ventricular tachycardia in humans. J Am Coll Cardiol 1988; 11:775-782.

Morady F, Kadish A, Rosenheck S, Calkins H, et al: Concealed entrainment as a guide for catheter ablation of ventricular tachycardia in patients with prior myocardial infarction. J Am Coll Cardiol 1991;17:678689.

Podczeck A, Borgreffe M, Martinez-Rubio A, Breithardt G: Termination of re-entrant ventricular tachycardia by subthreshold stimulus applied to the zone of slow conduction. Eur Heart J 1988;9:11461150.

Ruffy R: Termination of ventricular tachycardia by nonpropagated local depolarization: Further observations on entrainment of ventricular tachycardia from an area of slow conduction. PACE 1990;13:852-858.

Ruffy R, Friday KJ, Southworth WF: Termination of ventricular tachycardia by single extrastimulation during the ventricular effective refractory period. Circulation 1983;67:457-459.

Stevenson WG, Weiss J, Wiener I, Wohlgelernter D, et al: Localization of slow conduction in a ventricular tachycardia circuit: Implications for catheter ablation. Am Heart J 1987;114:1253-1258.

Waldo AL, Biblo LA, Carlson MD: Clinical importance of entrainment. J Cardiovasc Electrophysiol 1990;1:543-557.

Wellens HJJ, Durrer DR, Lie KI: Observations on mechanisms of ventricular tachycardia in man. Circulation 1976;54:237-246. 
This document is a scanned copy of a printed document. No warranty is given about the accuracy of the copy. Users should refer to the original published version of the material. 